The other paper describes a region in the carboxy-terminal tail of UNC93B 1 in which mutations enhance TLR7 responses without affecting trafficking to endosomes or TLR3 or TLR9 responses. Using a PKP to AAA mutation at residues 530-532 (UNC93B1-PKP/AAA), the authors identified syntenin 1 as a potential negative regulator of TLR7 signalling that interacts with wild-type UNC93B1 but not UNC93B1-PKP/AAA. Syntenin 1 is involved in the sorting of transmembrane receptors into intralumenal vesicles (ILVs), which can terminate receptor signalling. Indeed, UNC93B1 and TLR7 were enriched in exosomes expressing ILV markers purified from macrophage supernatant. Furthermore, ubiquitylation of UNC93B1-PKP/AAA, which is required for sorting into ILVs, was reduced compared with wild-type UNC93B1; the authors identified Lys333 as a key ubiquitylated residue, with inhibitory effects on TLR7, but not TLR3 or TLR9, signalling. They also identified UNC93B1 phosphorylation at

signalling was reported by both groups to promote $\mathrm{Nr} 1 \mathrm{~h} 3$ and Spic induction in monocytes, and Sakai et al. showed that this involves the engagement of RBPJ transcription factors. Furthermore, both studies identified crucial roles for transforming growth factor- $\beta$ (TGF $\beta$ ) and bone morphogenetic protein (BMP) signalling through SMAD4 in KC lineage commitment. Bonnardel et al. found that the production of colony-stimulating factor 1 (CSF1), TGF $\beta$ and BMPs by LSECs and stellate cells supports the induction of KC-associated genes in monocytes, and experiments by Sakai et al. in which SMAD4 was specifically deleted from KCs indicated an essential in vivo role for SMAD4 in KC development. Additional experiments by Bonnardel et al. showed that hepatocytes also contribute to shaping KC identity, as co-culture with these cells promoted the induction of Id3 in monocytes.

Detailed epigenetic analyses by Sakai et al. suggested that environmental signals in the liver act on pre-existing poised regulatory elements in recruited monocytes to rapidly induce transcription factors that specify KC identity and suppress monocyte-specific genes.
Ser457 and/or Ser550 as being required for binding of syntenin 1 and negative regulation of TLR7 signalling.

Interaction between UNC93B1 and syntenin 1 occurred at basal levels in unstimulated cells but was increased upon TLR7 signalling, which suggests a mechanism to both regulate the activation threshold for TLR7 and initiate a negative-feedback loop. Introducing Unc $93 b 1^{\text {PKP/AAA }}$ into the mouse germ line caused severe runting with systemic inflammation and autoimmunity that could be rescued by lack of TLR7.

Thus, these papers provide two new mechanisms by which UNC93B1 differentially regulates signalling by endosomal TLRs, through preventing ligand binding to TLR9 or by recruiting syntenin 1 for the downregulation of TLR7.

Kirsty Minton

ORIGINAL ARTICLE Majer, O. et al. Release from UNC93B1 reinforces the compartmentalized activation of select TLRs. Nature https://doi.org/ 10.1038/s41586-019-1611-7 (2019) | Majer, O. et al. UNC93B1 recruits syntenin-1 to dampen TLR7 signalling and prevent autoimmunity. Nature https://doi.org/10.1038/s41586-019-1612-6 (2019)

As $L X R a$ responds to changes in sterol and oxysterol concentrations, they measured the levels of these lipids in the liver and identified desmosterol as a potential hepatocyte-derived LXR agonist that may combine with DLL4 and the TGF $\beta / B M P$ pathway to induce KC lineage commitment.

Together, these studies detail the intricate cellular and molecular interactions that enable KC specialization and suggest a two-step model in which LXRa promotes initial commitment to the $\mathrm{KC}$ lineage and other transcription factors subsequently establish KC identity. These papers may serve as a blueprint to understand how common macrophage progenitors can become highly adapted to specific tissue microenvironments.

\section{Yvonne Bordon}

ORIGINAL ARTICLES Bonnardel, J., T'Jonck, W. et al. Stellate cells, hepatocytes, and endothelial cells imprint the Kupffer cell identity on monocytes colonizing the liver macrophage niche. Immunity https://doi.org/10.1016/j.immuni.2019.08.017 (2019)|Sakai, M., Troutman, T. D., Seidman, J. S. et al. Liver-derived signals sequentially reprogram myeloid enhancers to initiate and maintain Kupffer cell identity. Immunity https:// doi.org/10.1016/j.immuni.2019.09.002 (2019)

\section{Journal club}

\section{SUN-IMMUNE CONNECTION}

Exposure to ultraviolet radiation (UVR) damages skin cell DNA but skin cancers develop because UVR also affects the immune system. How does this occur? In 1983, De Fabo and Noonan identified a unique photoreceptor in skin urocanic acid (UCA) - that is an important intermediary by which UV wavelengths of 250-320 nm lead to systemic immunosuppression. Importantly, the characteristics of UVR-induced skin tumour development matched those of UVR-mediated reduction in contact hypersensitivity (CHS) responses in mice with an intact epidermis. Furthermore, the doseresponse relationship between UVR exposure and reduced CHS responses was very similar to the absorption spectrum of UCA, a major component of the epidermis, which isomerizes from the trans to the cis form upon irradiation.

Once cis-UCA had been identified as a photoreceptor, research on its biochemical properties intensified. Many cells of the skin (keratinocytes,

UCA, a major
component of
the epidermis ...
isomerizes from
the trans to the
cis form upon
irradiation


fibroblasts, dendritic cells, nerve cells and mast cells) and in the circulation (monocytes) can initiate signalling in response to cis-UCA, but its receptor in humans has been elusive; in mouse skin, it is the $5-\mathrm{HT}_{2 \mathrm{~A}}$ receptor. Cis-UCA has been detected in serum and urine following UVR exposure, which may explain some of the systemic immunoregulatory effects induced by UVR. Recently, increased levels of UCA in the blood and brains of mice exposed to moderate levels of UVR were implicated in enhanced learning and memory (Zhu et al., 2018).

There are other UVR photoreceptors in skin, including 7-dehydrocholesterol, DNA, membrane phospholipids and tryptophan, but the in vivo action spectrum of UCA discovered by De Fabo and Noonan showed that these other candidate molecules are not the major UVR photoreceptors for inducing immunosuppression. Instead, they may have a co-operative role with trans-UCA, with their involvement determined by the UVR wavelengths to which the skin is exposed, the immune pathways under investigation and whether the experiments are carried out in humans or animal models. As UVR is such an important component of our environment, this is a rich area for research.

Prue H. Hart Telethon Kids Institute, University of Western Australia, Perth, Australia e-mail:Prue.Hart@telethonkids.org.au

$$
\text { The author declares no competing interests. }
$$

ORIGINAL ARTICLE De Fabo, E. C. \& Noonan, F. P. Mechanism of immune suppression by ultraviolet irradiation in vivo. I. Evidence for the existence of a unique photoreceptor in skin and its role in photoimmunology.J. Exp. Med. 158, 84-98 (1983) RELATED ARTICLE Zhu, $\mathrm{H}$. et al. Moderate UV exposure enhances learning and memory by promoting a novel glutamate biosynthetic pathway in the brain. Cell 173 , 1716-1727 (2018) 